\title{
Senkung moderater Blutdruckwerte schützt nicht vor dem ersten Schlaganfall
}

Fragestellung: Ist eine blutdrucksenkende Therapie auch bei Personen mit einem mittleren kardiovaskulären Risiko ohne manifeste Herz-Kreislauf-Erkrankungen wirksam?

Hintergrund: Die arterielle Hypertonie ist populationsbezogen neben Vorhofflimmern der wichtigste Risikofaktor für den Schlaganfall. Dies gilt sowohl für zerebrale Ischämien als auch in noch höherem Umfang für zerebrale Blutungen.

Zweifelsfrei belegt ist der Nutzen einer aggressiven antihypertensiven Therapie nach einem ischämischen Insult oder einer Hirnblutung. Letztendlich nicht geklärt ist, wie aggressiv der Blutdruck bei Patienten mit mittlerem Risiko ohne bisherige kardiovaskuläre Ereignisse reduziert werden soll. Diese Frage sollte in der HOPE-3-Studie beantwortet werden.

Patienten und Methodik: Bei der hier vorliegenden Untersuchung handelte sich um eine randomisierte placebokontrollierte Doppelblindstudie. Eingeschlossen wurden 12.705 Teilnehmer mit einem mittelhohen kardiovaskulären Risiko, die bisher noch kein kardiovaskuläres Ereignis erlitten hatten.

Die Studienteilnehmer erhielten entweder täglich 16 mg Candesartan plus 12,5 mg Hydrochlorothiazid oder Placebo. Der primäre Endpunkt war die Kombination aus Tod aufgrund kardiovaskulärer Ursachen,

Lonn EM, Bosch J, López-Jaramillo $P$ et al. Blood-pressure lowering in intermediate-risk persons without cardiovascular disease. N Engl J Med 2016; 374: $2009-20$ nicht tödlichem Herzinfarkt und Schlaganfall. Der sekundäre Endpunkt schloss zusätzlich Herzstillstand, Herzinsuffizienz und operative oder interventionelle Revaskularisationen ein.
Ergebnisse: Die Patienten waren im Schnitt 65 Jahre alt und $45 \%$ waren Frauen. Die Risikofaktoren waren in abnehmender Häufigkeit Übergewicht, niedriges HDL-Cholesterin, arterielle Hypertonie, Rauchen sowie eine positive Familienanamnese für eine koronare Herzerkrankung (KHK). Der mittlere Beobachtungszeitraum betrug 5,6 Jahre.

Bei Studieneinschluss betrug der systolische Blutdruck in beiden Gruppen $138 \mathrm{~mm} \mathrm{Hg}$. In der aktiven Behandlungsgruppe nahm der systolische Blutdruck im Mittel um $10 \mathrm{~mm} \mathrm{Hg} \mathrm{ab,}$ in der Placebogruppe im Mittel um $4 \mathrm{~mm} \mathrm{Hg}$. Für die diastolischen Blutdruckwerte ergab sich ein Unterschied von $3 \mathrm{~mm} \mathrm{Hg}$ gegenüber Studienbeginn.

Im Hinblick auf die Häufigkeit des primären Outcomes ergaben sich keine Unterschiede: In der aktiven Behandlungsgruppe trat der primäre Endpunkt bei 4,1\% der Patienten auf (260 von 6.356) und in der Placebogruppe bei 4,4\% der Patienten (279 von 6.349).

Auch für den sekundären Endpunkt ergaben sich keine Unterschiede. Dasselbe galt für die Häufigkeit von Schlaganfällen und alle anderen vaskulären Endpunkte. Die Mortalität war mit $5,4 \%$ versus $5,5 \%$ ebenfalls nicht unterschiedlich zwischen den beiden Gruppen. Eine Subgruppenanalyse zeigte allerdings, dass diejenigen Patienten, bei denen der initiale Blutdruck oberhalb von $144 \mathrm{~mm} \mathrm{Hg} \mathrm{lag,} \mathrm{von} \mathrm{der} \mathrm{moderaten} \mathrm{Blutdrucksenkung}$ profitierten.

Schlussfolgerungen: Bei Personen mit einem mittleren kardiovaskulären Risiko, die bisher noch kein kardiovaskuläres Ereignis erlitten haben, führt eine moderate Blutdrucksenkung mit Candesartan und Hydrochlorothiazid nicht zu einer Reduktion von vaskulären Ereignissen.

\section{- Kommentar von Hans-Christoph Diener, Essen}

\section{Senkung höherer Blutdruckwerte und Sekundärprävention fokussieren}

\begin{abstract}
Bisher gilt bei Patienten mit arterieller Hypertonie eine anzustrebende systolische Blutdruckgrenze von $140 \mathrm{~mm} \mathrm{Hg}$, wenn bisher keine vaskulären Ereignisse aufgetreten sind, und von $130 \mathrm{~mm} \mathrm{Hg}$ für Diabetiker und für Patienten, die bereits ein vaskuläres Ereignis erlitten haben. Die hier vorliegende Studie zeigt, dass in der Primärprävention bei Personen mit moderaten systolischen Blutdruckwerten von im Mittel $138 \mathrm{~mm} \mathrm{Hg}$ eine Senkung des systolischen Blutdrucks um $8 \mathrm{~mm} \mathrm{Hg}$ vaskuläre Ereignisse über einen Zeitraum von fünf Jahren nicht reduziert. Daher sollten unsere therapeutischen Bemühungen auf Patienten konzentriert werden, bei denen die systolischen Blutdruckwerte über $140 \mathrm{~mm} \mathrm{Hg}$ liegen und insbesondere auf diejenigen Patienten, die bereits einen vaskulären Endpunkt erlitten haben.
\end{abstract}

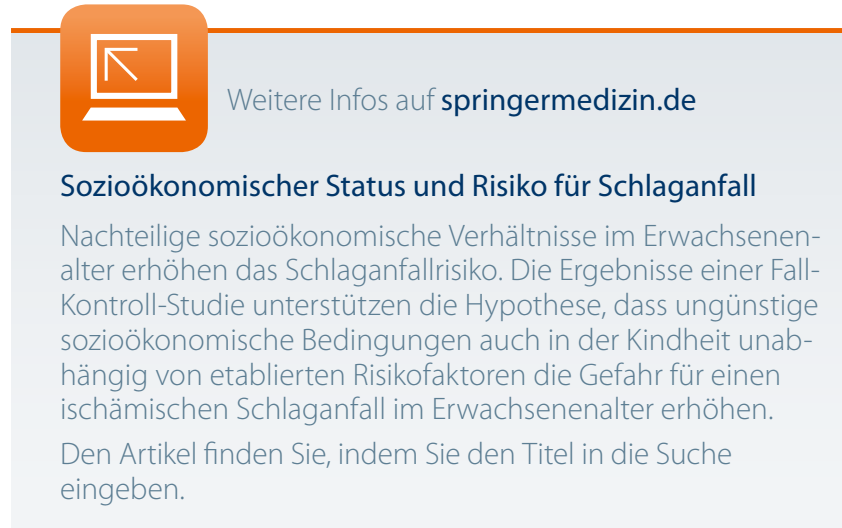

